\title{
Stories, voices, and explanations: How qualitative methods may help augment emergency medicine research
}

\author{
Patrick McLane, MA, PhD*'; Teresa Chan, MD, MHPE ${ }^{\ddagger \S \uparrow}$
}

\section{INTRODUCTION}

The use of qualitative methods in medical research has been the source of much controversy. To understand this, one needs only to read the (in)famous editorial written in 2015 on the British Medical Fournal (BM7) blanket policy of rejecting qualitative research. ${ }^{1}$ Trish Greenhalgh and colleagues were so compelling that they convinced the $B M 7$ editorial board to reconsider. ${ }^{2}$ As the response letter from the $B M F$ editors states: "Some research questions can only be answered by using qualitative methods."

In this issue of $C F E M$, we feature a paper about the role of paramedics in collaborative emergency centres that explores interprofessionalism, paramedic professional identity, and leadership. ${ }^{3}$ It demonstrates the importance of using qualitative methods to explore a program in an open-ended way, where quantitative methods would be limited to examining factors that researchers already believe to be relevant to the evaluation.

This editorial aims to introduce qualitative research to emergency medicine (EM) physicians. It describes some of the common characteristics of qualitative research, speaks to what qualitative research methods can and cannot do, and discusses some of the criteria for rigour in qualitative research.

\section{Characteristics of qualitative research}

Robert Yin describes five common characteristics of qualitative research. Qualitative research 1) explores the meaning that people attribute to experiences, 2) reports on the views and perspectives of the people studied, 3) takes into account the context studied, 4) contributes insights to existing or emerging concepts, and often 5) uses multiple sources of non-numerical evidence. ${ }^{4}$ Qualitative research may seek to better understand phenomena such as service delivery, site culture, patient experience, provider practice, and disease processes.

Of course, there are also questions for which qualitative research is unsuited. Qualitative research does not quantify a phenomenon or population, and so it does not reveal insights into prevalence of disease or commonness of certain problems. Although qualitative research can shed light on bow something might have worked, or why it did not work, a qualitative study cannot measure impact.

\section{Where qualitative research can fit in the Canadian EM context}

There is a substantive role for qualitative work in $\mathrm{EM},{ }^{5,6}$ and there are a number of opportunities to specifically highlight Canadian stories, Canadian voices, and explain phenomena within the world of Canadian EM.

New stories can be revealed by collecting data with key stakeholders within new contexts. A powerful example of this is a recent paper illuminating the experiences of bystanders after they have to perform cardiopulmonary resuscitation $(\mathrm{CPR})^{7}$; these stories may very well inform how we could redesign CPR courses to better prepare lay providers for action.

New voices can be heard from traditionally underrepresented or marginalized populations - from

From the ${ }^{*}$ Emergency Strategic Clinical Network ${ }^{\mathrm{TM}}$, Alberta Health Services; ${ }^{\dagger}$ Department of Emergency Medicine, University of Alberta, Edmonton, AB; $¥$ Division of Emergency Medicine, Department of Medicine; §McMaster Program for Education Research, Innovation, and Theory (MERIT); and IClinician Educator Area of Focused Competency (AFC) Training Program, McMaster University, Hamilton, ON.

Correspondence to: Patrick McLane, 2nd floor, South Tower, 7th Street Plaza, 10030107 St NW, Edmonton, AB, T5J 3E4, Canada; Email: mclane@ualberta.ca 
indigenous populations to transgendered youth, these are patients who have not been traditionally heard but should be listened to.

New explanations for phenomena of interest can also be generated through qualitative research. For instance, qualitative research can explore the factors impacting decision-making, as in a clinical context, or explore why a local implementation succeeds or fails.

There are a number of established traditions within qualitative research, which cannot be discussed in detail here, but which include phenomenology, narrative analysis, ethnography, and participant observation. Within the health sciences, grounded theory is frequently used. In a recent paper in March 2018, within this journal, Chan and colleagues provide a useful summary of qualitative methods and examples of each. ${ }^{8}$ Qualitative traditions are generally learned through graduate research training and have implications for what kind of data are collected and how they are analysed.

\section{Credibility in qualitative methods}

Credibility of qualitative research is generally demonstrated by investigators by 1) basing their work in theory and previous research; 2) using appropriate sampling and methods; 3) putting in the time to understand the context that they are studying; 4) handling data with respect and reflexivity (reflecting on how their personal experiences and biases might have influenced their findings); 5) using multiple sources of data to triangulate a phenomenon; 6) searching for and reporting evidence that might contradict their findings; and 7) striving for logical coherence. ${ }^{9,10}$ Chan and colleagues provide more literature on this topic. ${ }^{8}$

\section{Future of qualitative research in EM}

In many fields, the divide between quantitative and qualitative methods has begun to close. Many current graduate research methodology courses integrate both qualitative and quantitative methods. Newly minted $\mathrm{PhDs}$ are often expected to have a firm sense of the markers of rigour for all styles of research relevant to their field, and to have wrestled with the epistemological and ontological underpinnings of these styles of research. Indeed, the field of mixed methods has developed a robust working practice of integrating quantitative and qualitative methods, with a corresponding elaboration of the understandings of science that support this practice.
In the social sciences, where qualitative methods are well developed, practitioners have many tools with which quantitatively trained researchers may be unfamiliar. Depending on one's topic, it may be prudent to venture across campus for potential partners in research.

Great science is driven by great questions, and not all questions are best answered with quantitative methods. If we are beholden to a restrictive understanding of science based on positivist methodologies, then scientists in our field may be failing to recognize much of the science that we could be conducting. As the old saying goes: "If all you have is a hammer, everything looks like a nail." But not all clinical problems are nails. We hope that CFEM can provide a venue for qualitative work that highlights the stories, voices, and issues relevant to Canadian emergency clinicians.

Keywords: qualitative methods, research

Competing interests: None declared.

\section{REFERENCES}

1. Greenhalgh T, Annandale E, Ashcroft R, et al. An open letter to the $B M \mathcal{F}$ editors on qualitative research. $B M \mathcal{F}$ 2016;352(2):1-4; doi:10.1136/bmj.i563.

2. Loder E, Groves T, Schroter S, et al. Qualitative research and the BMF. BMF 2016;352(2):1-2; doi:10.1136/bmj.i641.

3. Whalen $\mathrm{S}$. The novel role of paramedics in collaborative emergency centres aligns with their professional identity: a qualitative analysis. Can 7 Emerg Med 2018;20(4); doi:10.1017/cem.2018.401.

4. Yin RK. Qualitative research from start to finish, 2 nd ed. New York: Guilford Press; 2010.

5. Choo EK, Garro AC, Ranney ML, et al. Qualitative research in emergency care part I: research principles and common applications. Acad Emerg Med 2015;22(9):1096102; doi:10.1111/acem.12736.

6. Ranney ML, Meisel ZF, Choo EK, et al. Interview-based qualitative research in emergency care part II: data collection, analysis and results reporting. Acad Emerg Med 2015;22(9):1103-12; doi:10.1111/acem.12735.

7. Mausz J, Snobelen P, Tavares W. "Please. Don't. Die". Circ Cardiovasc Qual Outcomes 2018;11(2):e004035; doi:10.1161/ CIRCOUTCOMES.117.004035.

8. Chan TM, Ting DK, Hall AK, et al. A writer's guide to education scholarship: qualitative education scholarship (part 2). CFEM 2018;20(2):284-92; doi:10.1017/cem.2017.25.

9. Guba EG. Criteria for assessing the trustworthiness of naturalistic inquiries. Educ Commun Technol 1981;29(2):7591; doi:10.1007/BF02766777.

10. Shenton AK. Strategies for ensuring trustworthiness in qualitative research projects. Educ Inf 2004;22(2):63-75; doi:10.3233/EFI-2004-22201. 